\title{
Suppression of the Coffee-Ring Effect by Tailoring the Viscosity of Pharmaceutical
}

\section{Sessile Drops}

\author{
Janine Wilkinson $^{\mathrm{a}}$, Chak Tam ${ }^{\mathrm{a}}$, Alexandros Askounis ${ }^{\mathrm{b} *}$ and Sheng Qi ${ }^{\mathrm{a}^{*}}$

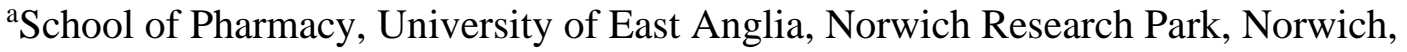 \\ NR4 7TJ \\ ${ }^{\mathrm{b}} \mathrm{School}$ of Engineering, University of East Anglia, Norwich Research Park, Norwich, \\ NR4 7TJ
}

Corresponding authors: Dr Sheng Qi, sheng.qi@uea.ac.uk; Dr Alexandros Askounis, a.askounis@uea.ac.uk

The authors have no conflict of interest 


\begin{abstract}
The coffee ring effect (CRE) lies at the heart of droplet-based processing techniques where the drying process of the solute-laden droplet leads to the undesirable formation of a ring-like pattern with uneven distribution of the solidified solute. Although the CRE has been well studied, the practical strategies reported in the literature to prevent CRE are still limited. In this study, we aimed to develop a simple practical solution to minimise CRE by adding polymeric excipients to pharmaceutical solutions containing active pharmaceutical ingredients. We investigated the influence of viscosity on multicomponent sessile droplets containing a polymer (chitosan) and a model drug (paracetamol). When dried on glass, a sessile droplet of an aqueous paracetamol solution was shown to recrystallise into an undesired coffee-ring pattern of drug crystals, due to CRE. This peripheral deposition of crystalline solute was suppressed when the formulation was thickened with chitosan. Increasing the viscosity of the solution prevented the CRE by immobilising solute within the droplet, preventing the radial flow of the solute to the edge of the droplet. This study enriches the mechanistic understanding of a simple practical solution to the suppression of CRE which can have wide applications in many industrial sectors including pharmaceutical.
\end{abstract}

\title{
Key Words
}

Coffee ring effect, chitosan, solid dispersion, droplet casting, drying suspensions, paracetamol, 


\section{Introduction}

The coffee ring effect (CRE), as described by Deegan and colleagues in their seminal work [1] and work thereafter $[2,3]$, explains that during the evaporation of a sessile droplet containing solute, the droplet is pinned and then liquid is lost at the contact line. In order to replenish this loss, liquid flows radially from the centre of the droplet to the contact line by capillary flow, carrying solute along with it and depositing it around the edge of the droplet. The CRE not only causes heterogeneity in the solute distribution after drying but also undesired physical forms changes, such as recrystallisation of the solute in the coffee-ring deposits $[4,5]$. Therefore, for manufacturing processes involving evaporation of droplets on surfaces, such as inkjet printing, CRE can have significant practical implications on the quality of the end products [6]. When such manufacturing methods are used for the production of diagnostic and microdot arrays [7], electronics [8], and recently pharmaceuticals $[9,10]$, the homogeneity of the distribution of the active ingredients in the dried film formed from the droplet is often critical to the performance of the final dried product $[11,12]$.

Investigating the microstructure of the CRE deposits can contribute to the strategy development of how to reduce or even suppress the effect. To prevent the CRE, three main theoretical strategies have been proposed in the literature: 1) to avoid pinning of the contact line, 2) curbing the capillary flow of liquid to the contact line and 3) to prevent solid particles from moving to the contact line [13]. Practical examples of reducing and preventing CRE include changing the shape of the solute [14], manipulating the surface of the substrate [15], addition of sugars and salts to the solution $[16,17]$ and the addition of surfactants [18].

Viscosity describes a fluid resistance to flow and as such by increasing the viscosity, the radial flow inside a droplet will be hindered thereby reducing the migration of the solute to the edge of the droplet and preventing the CRE. The effect that viscosity has on the CRE has been discussed in literature. An example of this by Fredrich et al. [11] demonstrated that addition of a viscosity enhancing binder agent, ethyl cellulose, to an ink will significantly reduce the severity of the CRE. In another inkjet study, Talbot [19] used Laponite to induce a sol-gel transition of the ink and discovered that Laponite supressed radial flow and produced a uniform deposit. Research by Cui et al [20] explains further that when using hydrosoluble polymers to increase the viscosity of a droplet containing $\mathrm{SiO}_{2}$ microspheres, the constant motion of the contact line (de-pinning) allowed a homogenous deposit to be left after complete drying and the reduction of solid particles on the contact line. The results of these studies indicate that 
adjusting the viscosity of a solution can be used as a practical tool to control solute deposition and prevent CRE. However, this has not been studied in a pharmaceutical context, in which multiple types of solutes (including the active pharmaceutical ingredient and multiple excipients) are often included.

The aim of this study is to investigate the effect of viscosity on the suppression of CRE of a model pharmaceutical system with an attempt to offer formulation strategies to prevent CRE in the manufacturing of pharmaceutical products involving droplet drying. In this study, paracetamol, being used as the model active pharmaceutical ingredient was combined with different concentrations of chitosan, a polymeric pharmaceutical excipient that is often used as a viscosity enhancer to provide mucoadhesion, to promote tight junction opening and for its anti-microbial effects[21]. This drug-polymer combination created a viscosity-based multicomponent approach to suppress CRE. The results of this study provide new insights into the fundamental importance of CRE on the quality of drug delivery products manufactured using methods involving droplet drying for pharmaceutical applications and importantly, how to reduce the CRE. This is the first study, to the best of our knowledge, to reveal the fundamental dynamics of how pharmaceutical based multicomponent droplets dry to form a film and how one may reduce the coffee ring effect in these products.

\section{Experimental}

\subsection{Materials}

Paracetamol (Acetaminophen) and medium molecular weight chitosan (75-80\% deacetylated) were both purchased from Sigma-Aldrich (St. Louis, MO, USA). Glacial acetic acid was purchased from ThermoFisher Scientific (MA, USA).

\subsection{Preparation of solutions}

Table 1 shows the composition and properties of the solutions used in the study. The solutions were prepared by mixing the dry powder ingredients (paracetamol and chitosan) and liquid (dilute acetic acid) at room temperature with a magnetic stirrer (at $800 \mathrm{rpm}$ ) for four hours. The formulations were denoted with the letter $\mathrm{F}$ followed by a number relating to the $\%(\mathrm{w} / \mathrm{w})$ concentration of chitosan diluted in $2 \%(\mathrm{v} / \mathrm{v})$ acetic acid aqueous solution. 
Table 1. The list of the compositions and basic physicochemical properties of the solutions studied

\begin{tabular}{|c|c|c|c|c|c|}
\hline $\begin{array}{l}\text { Formulation } \\
\text { Code }\end{array}$ & $\begin{array}{l}\text { Paracetamol } \\
(\% \mathrm{w} / \mathrm{w})\end{array}$ & $\begin{array}{l}\text { Chitosan } \\
(\% \text { w/w })\end{array}$ & $\begin{array}{l}2 \%(\mathrm{v} / \mathrm{v}) \text { Acetic } \\
\text { Acid in } \mathrm{H}_{2} \mathrm{O} \\
(\% \mathrm{w} / \mathrm{w})\end{array}$ & $\begin{array}{l}\text { Dynamic Viscosity } \\
(\mathrm{mPa} . \mathrm{l}]) \text { at } \\
\text { Shear } 40 \mathrm{y}(1 / \mathrm{s})\end{array}$ & $\begin{array}{l}\text { Surface Tension } \\
(\mathrm{mN} / \mathrm{m})\end{array}$ \\
\hline Fo & 0.2 & - & 99.8 & $6.2 \pm 0.2$ & $65.8 \pm 2.1$ \\
\hline F0.25 & 0.2 & 0.25 & 99.5 & $14.2 \pm 1.0$ & $66.2 \pm 1.4$ \\
\hline F0.5 & 0.2 & 0.5 & 99.3 & $28.8 \pm 2.3$ & $66.3 \pm 2.1$ \\
\hline F1.0 & 0.2 & 1 & 98.8 & $81.8 \pm 0.6$ & $65.4 \pm 1.2$ \\
\hline F2.0 & 0.2 & 2 & 97.8 & $398 \pm 60.8$ & $66.4 \pm 2.6$ \\
\hline
\end{tabular}

\subsection{Sessile Drop and Surface Tension Measurements}

The evaporation of a sessile droplet was followed using a sessile droplet analyser (DMS-401, Kyowa interfacial science, Japan). Three microliters of each solution were deposited onto a glass microscope slide and their evaporation was analysed using the instrument's dedicated FAMAS software. The contact angle (CA) and contact radius (CR) of the droplets over time were measured from the images within the video during drying $(n=9)$. The slide was maintained at $50^{\circ} \mathrm{C}$ with a heater element to emulate manufacturing conditions. The surrounding environmental conditions were monitored at $23^{\circ} \mathrm{C}$ and relative humidity $45 \pm 10 \%$. The surface tension $(\mathrm{mN} / \mathrm{m})$ of each solution was measured using the same equipment with the pendant drop function $(\mathrm{n}=10)$ and is shown in Table 1.

\subsection{Scanning Electron Microscopy (SEM) and Polarising Light Microscopy (PLM)}

SEM studies were carried out with a Gemini 300 series microscope (Zeiss, Germany), operating at $10 \mathrm{kV}$ acceleration voltage. Prior to SEM imaging, samples were sputter coated with $\mathrm{Au}$ for $30 \mathrm{sec}$ at $2.2 \mathrm{kV}$ and $20 \mathrm{~mA}$ at $55 \mathrm{~mm}$ (Polaron SC 7640, Quorum Technologies, UK). Light microscopy observation was carried out with a Leica DM LS2 microscope (Wetzlar, Germany) with a polarising filter. The images were collected using Studio Capture, Version 3.1.1 (Roland, UK).

\subsection{Confocal Microscopy}


Precise three dimensional topographical measurement and height profile extraction of the dried patterns were measured and processed with a 3D Measuring Laser Microscope (LEXT OLS5000, Olympus, Japan).

\subsection{Rheological measurements}

Dynamic viscosity measurements of each solution were measured on a Discovery Hybrid Rheometer (TA Instruments, Newcastle, DE, United States) using a $40 \mathrm{~mm}$ steel parallel plate across an increasing range of shear $(0.01-4001 / \mathrm{s})$ at $50^{\circ} \mathrm{C}$. Data was analysed using TRIOS software (TA Instruments, Newcastle, DE, USA).

\subsection{Surface roughness measurements using a contact profilometer}

The roughness of the glass slide used to deposit the sessile drops was measured using a Stylus Profilometer (DektakXT, Bruker, MA, USA). The glass slides $(n=2)$ were scanned using the 'Hills and Valleys' profile at left, right and middle positions with a stylus force of $1 \mathrm{mg}$ with a scan length of $2 \mathrm{~mm}$ (data not shown). The results of the glass slides show considerable roughness as evidenced as peaks and troughs in the profilometer scans of the surfaces with the root mean square roughness $(\mathrm{Pq})$ of $0.0205 \pm 0.0052 \mu \mathrm{m}$.

\section{Results and Discussion}

\subsection{Phases of Evaporation}

The drying process of the sessile drops were recorded and the images, as shown in Figure 1, were analysed in order to obtain kinetics information of the drying. During the evaporation of a sessile drop there are two parameters that were used to describe the kinetics, the contact radius (CR) and the contact angle (CA). Drying often can be observed in different phases when one of these parameters remains constant, identified as the constant contact radius (CCR) and the constant contact angle (CCA) phases [12, 22-24]. F0 is a $2 \% \mathrm{w} / \mathrm{w}$ acetic acid aqueous solution of paracetamol with no chitosan and acts as our baseline control sample for our viscosity studies. A droplet of this formulation completely dried after ca. 200 seconds leaving behind a coffee-ring pattern due to CRE (red arrow, Figure 2). In the first half of the drying time (0100 seconds), the droplet maintained a constant contact radius (CCR phase), also known as 'pinned droplet', while the CA constantly decreases, due to surface heterogeneities [24]. This observed roughness of the glass substrate surface measured by a profilometer facilitate the 
pinning effect of the droplet during drying [24] and contributed to the observed initial steady CCR phase. The pinned contact line resulted in radial flow from the centre of the drop to the periphery delivering solute to the edge, as evidenced by the reduction in the CA (Figure 2). The flow to the contact line, caused by capillary action [1-3], carried solute to the contact line where it accumulated and pinned the drop stronger. At 100 seconds evaporation enters the CCA regime, with moving contact line. In the final phase of drying 170-201 seconds, the droplet evaporated under a mixed mode whereby both CR and CA decrease in unison until drying is complete.

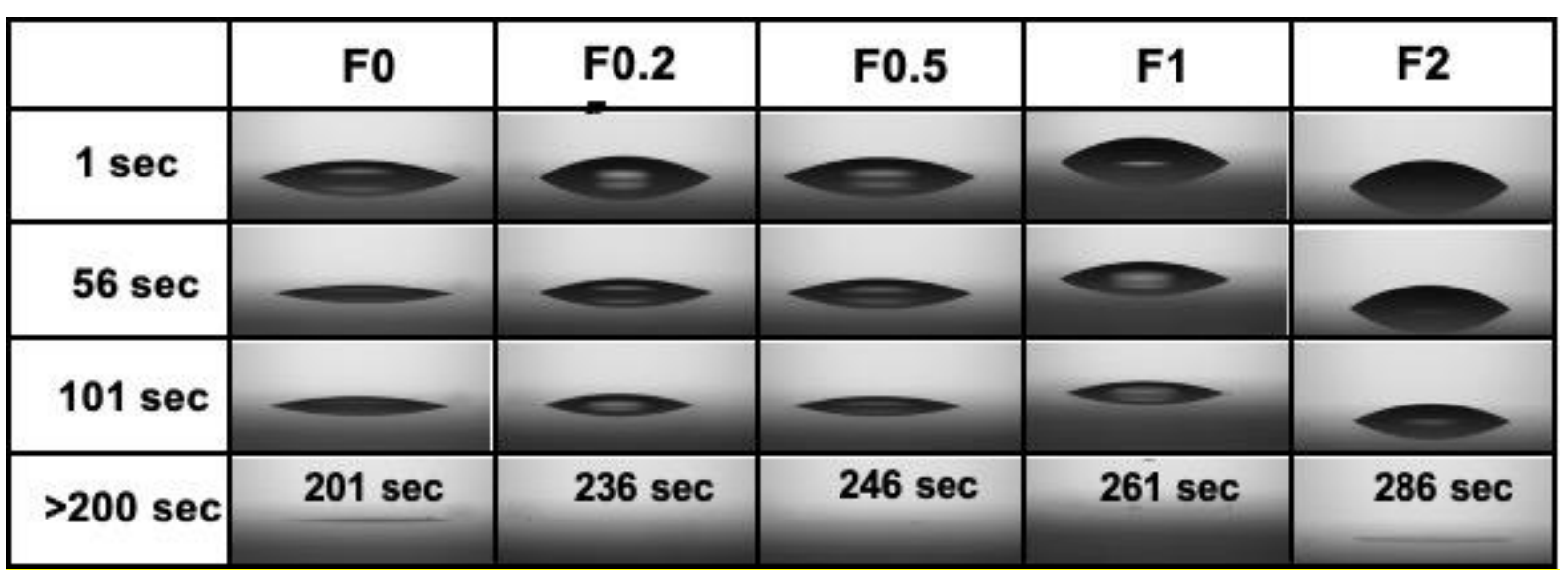

Figure 1. Snapshots of the evaporation evolution taken during the droplet drying of the formulations F0, F0.25, F0.5, F1, and F2 at 1 second, 56 seconds, 101 seconds until disappearance of the drop beyond 200 seconds (labelled individually for each formulation). 


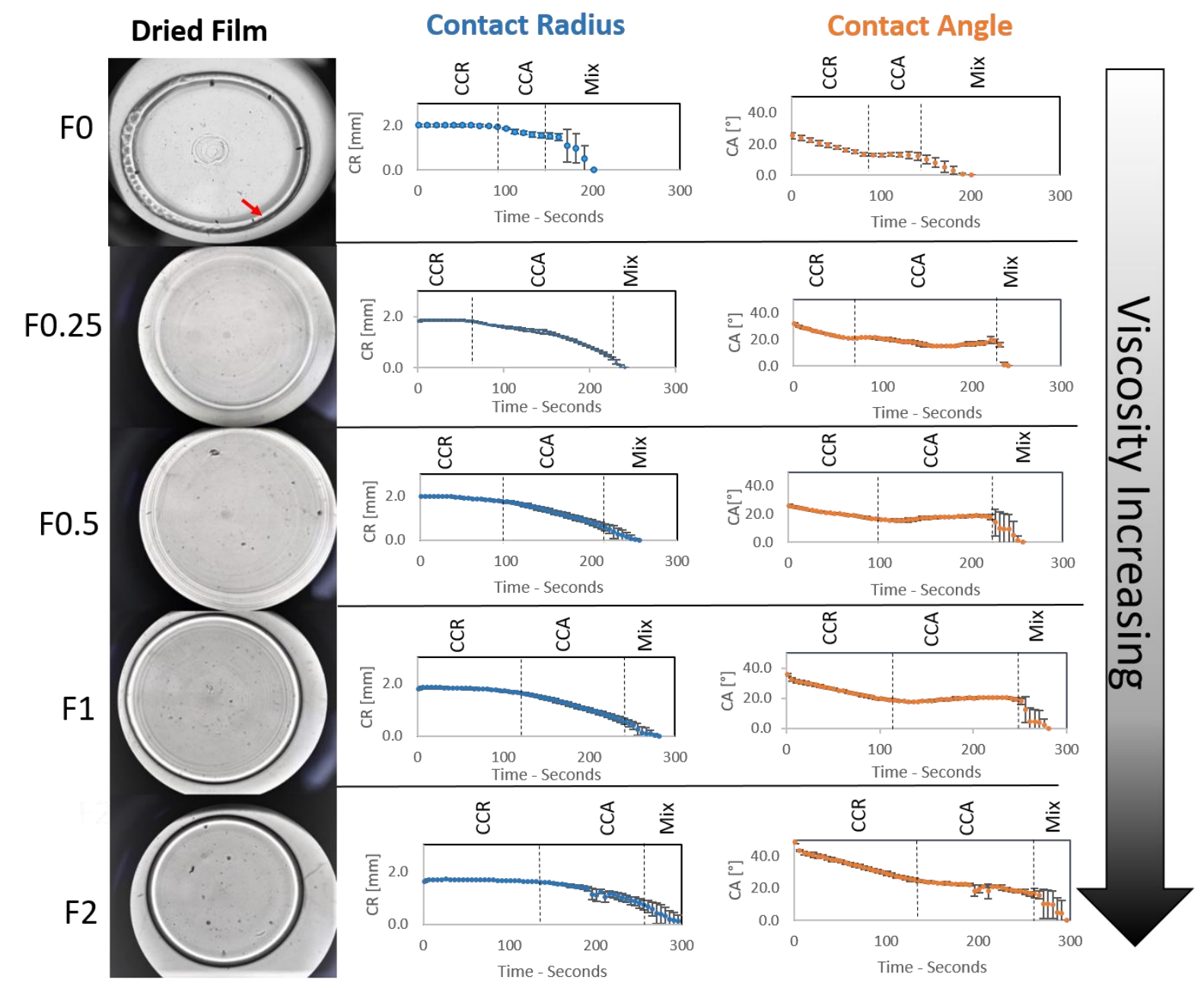

Figure 2. Evolution over time of $C R$ and contact angle $C A$ and representative light microscopy images of the dried films. A typical CRE (indicated by a red arrow) can be observed in the dried film of FO, but substantially supressed in the dried films containing chitosan.

The addition of $0.25 \% \mathrm{w} / \mathrm{w}$ chitosan in the solution (F0.25) more than doubled the solution's viscosity and led to a thinner CRE line at the periphery, as shown in Figure 2. The kinetics show that the CCR mode was shorter at 65 seconds and followed by a considerably longer CCA mode before the drop entered a much shorter mixed mode. These results indicate that the increase in viscosity has suppressed (although not completely due to the thin deposit) the outward radial flow [1]. It is noted that a small central area of deposition was observed, but this is not as discernible as seen in F0 and is due to the fact that the mixed phase is much shorted for F0.25 than for F0. 
Increasing the chitosan concentration to $0.5 \%$ further increased the viscosity of F0.5. This lengthened the amount of time the droplet spent in the CCR phase compared to F0.25 (Figure 2). This suggests that the contact line was more resistant to moving, but it is questionable if it has been 'pinned' or immobilised in the same way it was in the case of F0 or F0.25. To be truly pinned, enough solute must be deposited at the edge to facilitate immobilisation of the contact line [20]. However, the fact that no clear coffee-ring was discerned suggests that the radius stayed constant due the effect of viscosity. In the second half of the drying graph, the CA became constant while the radius decreased (CCA). It is during this period that the observed uniform deposition of the solute happened, as the contact line moved continuously, depositing homogeneously the solute while it retracted.

The final two solutions, F1 and F2 containing 1\% and 2\% chitosan, respectively, dried without resulting in a coffee ring (Figure 2). The drying kinetics for both formulations were similar to F0.5, except that the CCR mode and overall drying time extend as viscosity increased. Both F1 and F2 had much higher viscosity (Table 1) which caused the droplets themselves to be 'viscous bodies', an immobile drop that does not flow. Hence, the internal outward radial flow from the centre to the edge was considerably slower relative to F0 and F0.25. This means that solute within remained homogenously distributed within the droplet and upon drying results in a homogenous film.

Notably, CA increased from F0 to F2, suggesting a gradual transition to a more spherical droplet. To rule out the effect of the addition of increasing amount of chitosan on the surface tension of the solutions, we measured the surface tension of each solution and found similar values for all chitosan containing formulations F0.25 - F2 (Table 1). Rather, the gradual transition to a spherical drop with increasing chitosan content should be attributed to viscosity. This viscosity induced the no-spreading or "beading" of the droplet on top of the glass substrate. This is supported by the decreasing value of initial CR with increasing viscosity from F0 to F2. Essentially, the moment each droplet is gently deposited on the glass slide, it should start spreading until it reaches its equilibrium CA. However, viscosity inhibits the process resulting in the observed different initial CA. This "beading" of the droplet due to viscosity should also explain why the evaporation times became longer with viscosity, as thinner droplets tend to evaporate faster [25]. 


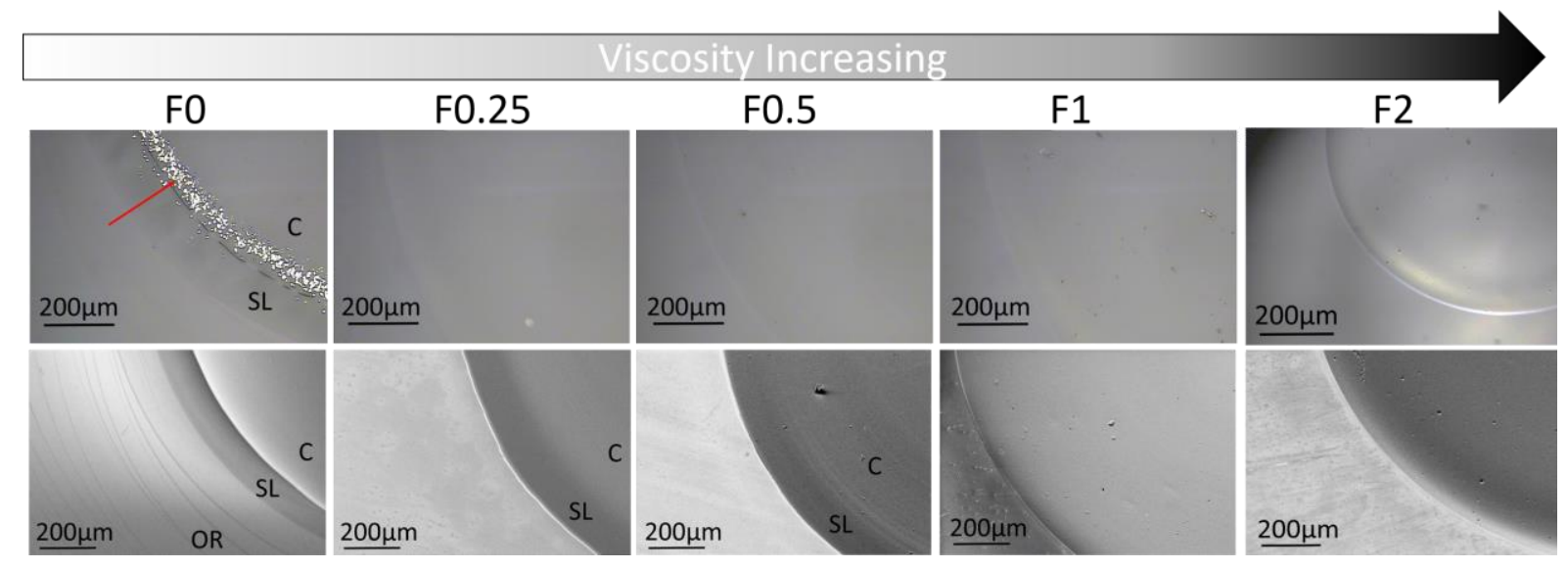

Figure 3. PLM (top row) and SEM (bottom row) images of the edges of the droplets. $C=$ Centre, $S L=$ Stagnation line, OR=outer rings. Crystalline drug deposits are only seen in FO.

\subsection{Location of crystallised solute}

The relevance of CRE in pharmaceutical applications is two-fold. Firstly, the uniform distribution of the active pharmaceutical ingredient across the deposited film after drying of a multi-component solution is one of the key quality attributes of the finished product. As demonstrated by the results of F0 formulation, CRE caused the uneven distribution of the drug crystals that were mainly concentrated at the edges of the dried films. Secondly, for most of the solid dispersion-based film products, the drug, in particular for poorly water-soluble drugs, is desirable to be molecularly dispersed within excipients (i.e. polymers) in the films. This could ensure the product to achieve the desired drug release performance and storage stability profile. Any crystallisation of the drug during drying could significantly reduce the drug dissolution rate and lower the storage stability of the product. In our case, paracetamol was added in the same concentration into each formulation. Locating the position of the recrystallised paracetamol within the dried deposits was carried out using polarised light microscopy (PLM). F0 was the only formulation with evidence of crystalline materials (seen as birefringence) (Figure 3). Under PLM, these crystalline drug particles were found only at the periphery of the F0 deposit, adjacent to it and centre-side (red arrow in F0 panel in Figure 3). The positioning of the crystals suggested that during drying, the uniformly distributed solute was moved and aligned by radial flow within the droplet to the contact line which has been pinned for long enough for the paracetamol to be deposited. However, what is clearly seen on the PLM and SEM images are the three parts to the deposit, the central region (C), the stagnation line (SL) and outer rings (OR). In the SEM images, the stagnation line shows up as 
a contrasting dark outer line, maybe due to a pinned contact line, and the outer rings are evidence of 'stick-slip' events.

The absence of any birefringence in the PLM images of F0.25 - F2, as seen in the top row of Figure 3, indicates that the addition of $0.25 \%$ chitosan inhibited the recrystallization of paracetamol. The SEM image (bottom row of Figure 3) shows that F0.25 has a stagnation line and is a similar width to the stagnation line in F0 of approximately $200 \mu \mathrm{m}$. There are no outer rings and therefore no evidence of 'stick-slip' events.

The SEM image of F0.5 shows no features in terms of stagnation line or outer rings and is more uniform (Figure 3). This suggests that F0.5 is the transition solution where the concentration of chitosan $(0.5 \%)$ is enough to create a homogeneous solution whilst drying. Similarly to F0.5, the film created by solution F1 shows uniformity morphology under SEM with a raised appearance across the film. F2 solution has the greatest viscosity amongst all tested solutions. After drying, the raised edge was further amplified in comparison to $\mathrm{F} 1$ and formed a uniform film with a concave form and round edges (Figure 3).

\subsection{Patterning of Films}

The confocal microscopic analysis was used to confirm the height profile of the edges of the films. Confocal microscopy also revealed detailed topography not shown in the SEM or optical light microscopy images. The results shown in Figure 4 further confirmed the SEM findings. F0 has a small raised edge at the periphery of the dried droplet but no patterning inside of this ring, with a thickness approximately $2 \mu \mathrm{m}$. When compared with the SEM image, it is likely that the crystalline deposits are located adjacent to and centre side of this ridge. Significant concentric ring patterns are observed in F0.25, F0.5, and F1, suggesting that the contact line was pinned and unpinned frequently throughout drying as described by others $[12,15,26]$. The thickness of the edges of these films increased to approximately $5 \mu \mathrm{m}$. It is interesting to note that the frequency of the ring pattern reduces as the chitosan concentration increases. This is due to the chitosan-enhanced viscosity reducing the radial flow within the drying droplet. The most viscous solution, F2, created minimal patterning during evaporation, commensurate with a gel-like substance that has not flowed during drying, but doubled the thickness of the edge in comparison to F1. Due to the relatively high concentrations of chitosan added to F1 and F2 formulations, there is more solid deposition left behind after the liquid has evaporated, thus a 
thickness of approximately 3 and $5 \mu \mathrm{m}$, respectively. In this case, the films of F1 and F2 are thicker, more uniform across the surface and leave a broad, rounded edge.

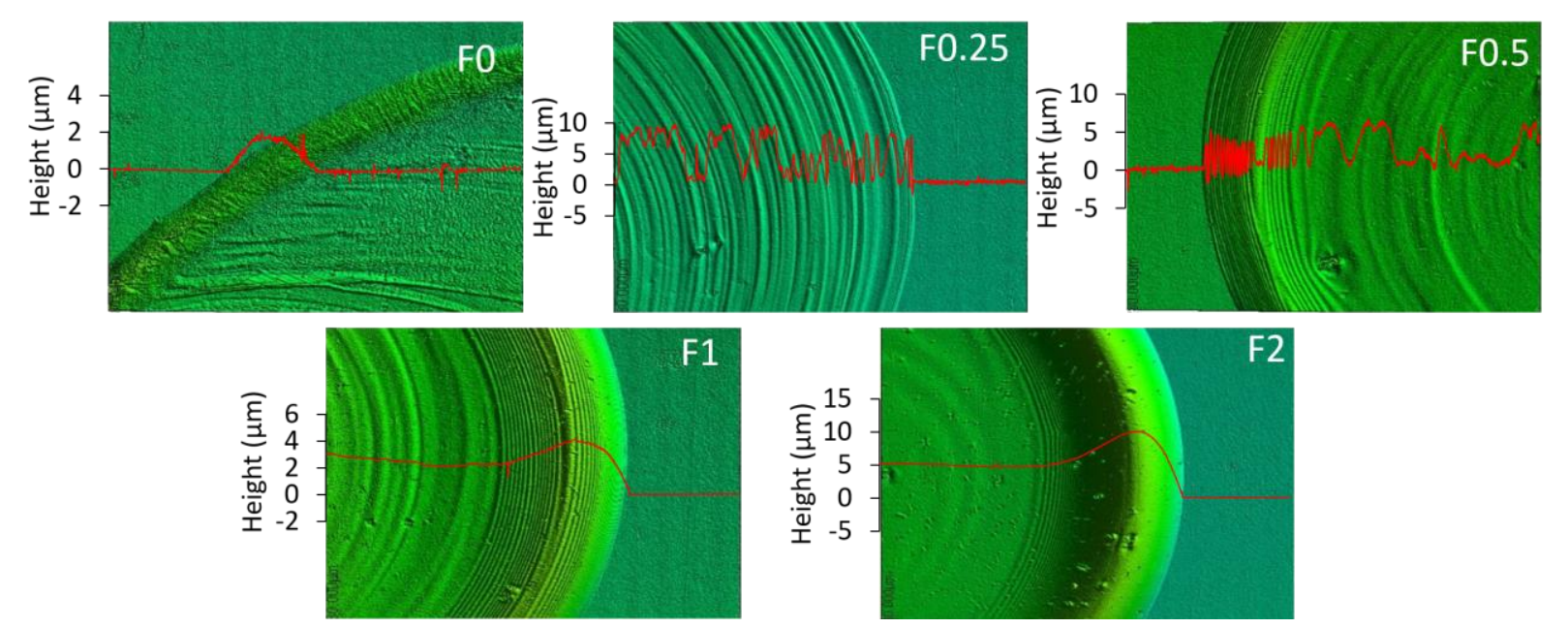

Figure 4. Confocal microscopy of multicomponent films. Profile trace (red) identifies deposition patterning across the surface of the film.

\subsection{CRE Suppression Mechanism}

Radial flow as described by Deegan and colleagues in 1997 [1] is driving the solute in an aqueous solution to the contact line by radial flow during evaporation (Figure 5a). In this study, this is the mechanism that facilitated the crystalline drug coffee ring that is observed in F0. The paracetamol molecules dissolved within the droplet moved to the edge of the drying drop. Due to the evaporation of liquid at this point, the concentration of paracetamol increased resulting in crystallisation as they exceed their saturation solubility (Figure 5b). With the addition of a small concentration of chitosan, we postulate that the radial flow was reduced because viscosity was introduced into the system (Figure 5c). The presence of the polymer improved the homogeneity of the drug molecule distribution within the colloidal droplet and reduced the nucleation tendency. This inhibited the drug recrystallization in the droplets containing chitosan, but a thin film of dried chitosan remained (Figure 5d). The addition of a higher concentration of chitosan (Figure 5e) created a droplet that acted as a viscous body and radial flow is inhibited. In these cases, the presence of chitosan again prevented the crystallisation of the drug by preventing critical mass which was required to form a nucleus, only a thick film of chitosan remained after evaporation (Figure 5f). 
a Strong Radial Flow

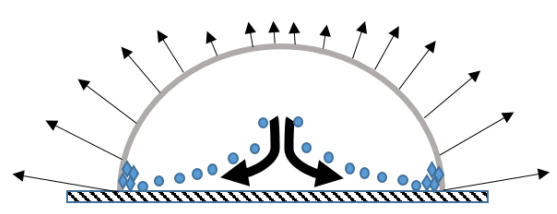

C Reduced Radial Flow

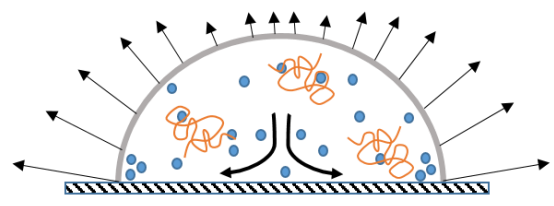

e Suppression of Radial Flow

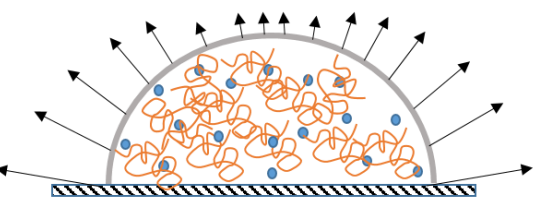

b Residues deposited

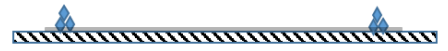

d Thin Dried Film

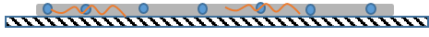

f Thick Dried Film

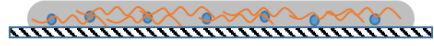

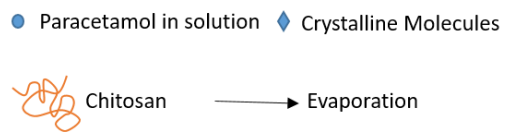

Figure 5. The mechanism driving the suppression of crystalline coffee-rings from a pharmaceutical multicomponent solution.

Furthermore, it is imperative to point out here that the multicomponent solutions in this study changed phase whilst drying, starting out as a liquid and terminating as a solid film. It is important to acknowledge that solutions possessing a 'phase change system' during evaporation will show a different pattern during drying compared to a simple suspension such as coffee [12].

Introducing viscosity provided resistance to the movement of fluid and solutes inside the droplet. In F0, solute was free to move radially from the centre of the droplet to the edge and was deposited but in the other chitosan containing solutions, the increased viscosity reduced radial flow, hindering the movement of the solute to the edge of the drying droplet (Figure 5e and f). Studies using polyethyleneoxide (PEO) to increase solution viscosity reported aberration from CRE. They too, explained this as a restriction to flow inside the droplet as observed in the current study [20]. Due to the now minimal solute being deposited at the edge of the droplet, the contact line remained mobile, retracting constantly towards the centre of the droplet [20] until drying was complete. 
Overall, F0.5 has the correct qualities to provide a uniformly dispersed pharmaceutical film. It provides a symmetrical, round droplet with enough viscosity to prevent the capillary flow of solutes to the contact line and also prevents pinning of the contact line. At higher concentrations of chitosan, there is no added advantage for processing. Droplets of F1 and F2 act as nonflowing viscous bodies. Although they inhibited the formation of CRE, they are more difficult to handle and required longer drying time due to their greater viscosity and pose challenges for scale-up production.

\section{Conclusion}

This study explored the use of viscosity as a means to suppress CRE in multicomponent pharmaceutical solutions. The data demonstrated that when paracetamol dried in a simple aqueous solution, paracetamol recrystallized and formed a coffee-ring structure. The addition of chitosan introduced viscosity into the system and prevented the recrystallization and the formation of coffee-ring of paracetamol around the edge of the film. This can be explained by the flow within the droplet being stalled within a viscous system which led to more homogeneous distribution of the solute during drying. F0.5 was found to be the optimal solution, as it left behind the evaporation a uniformly dispersed pharmaceutical film.

In this study, we provided two main contributions to understanding how to suppress CRE. Firstly, increasing solution viscosity can be successfully employed as a practical means to suppress the CRE to obtain a uniform film following drying of a multicomponent pharmaceutical droplet. Secondly, for the first time we report that once a suitable viscosity has been reached, further increases of the viscosity are not beneficial, because it increases the risk of obtaining non-uniform thickness of the film and prolongs the drying time. The methodology established herein for studying low volume sessile drops can be used as a rapid screening method to identify the optimal viscosity range of a formulation to ensure the homogeneous drying of films for a wide range of industrial applications, not limited to pharmaceuticals.

\section{Acknowledgement}

This project has received funding from the Interreg 2 Seas programme 2014-2020 co-funded by the European Regional Development Fund under subsidy contract 2S01-059_IMODE. 


\section{References}

1. Deegan, R.D., et al., Capillary flow as the cause of ring stains from dried liquid drops. Nature, 1997. 389(6653): p. 827-829.

2. Deegan, R.D., et al., Contact line deposits in an evaporating drop. Physical Review E, 2000. 62(1): p. 756-765.

3. Deegan, R.D., Pattern formation in drying drops. Physical Review E, 2000. 61(1): p. 475-485.

4. Askounis, A., et al., The effect of evaporation kinetics on nanoparticle structuring within contact line deposits of volatile drops. Colloids and Surfaces A: Physicochemical and Engineering Aspects, 2014. 441: p. 855-866.

5. Askounis, A., et al., Effect of particle geometry on triple line motion of nano-fluid drops and deposit nano-structuring. Advances in Colloid and Interface Science, 2015. 222: p. 44-57.

6. Park, J. and J. Moon, Control of Colloidal Particle Deposit Patterns within Picoliter Droplets Ejected by Ink-Jet Printing. Langmuir, 2006. 22(8): p. 3506-3513.

7. de Gans, B.J. and U.S. Schubert, Inkjet printing of well-defined polymer dots and arrays. Langmuir, 2004. 20(18): p. 7789-93.

8. Vaseem, M., et al., Inkjet printed fractal-connected electrodes with silver nanoparticle ink. ACS Appl Mater Interfaces, 2012. 4(6): p. 3300-7.

9. Boehm, R.D., et al., Inkjet printing for pharmaceutical applications. Materials Today, 2014. 17(5): p. 247-252.

10. Scoutaris, N., S. Ross, and D. Douroumis, Current Trends on Medical and Pharmaceutical Applications of Inkjet Printing Technology. Pharmaceutical Research, 2016. 33(8): p. 17991816.

11. Friederich, A., J.R. Binder, and W. Bauer, Rheological Control of the Coffee Stain Effect for Inkjet Printing of Ceramics. Journal of the American Ceramic Society, 2013. 96(7): p. 20932099.

12. Sefiane, K., Patterns from drying drops. Advances in Colloid and Interface Science, 2014. 206: p. 372-381.

13. Mampallil, D. and H.B. Eral, A review on suppression and utilization of the coffee-ring effect. Adv Colloid Interface Sci, 2018. 252: p. 38-54.

14. Yunker, P.J., et al., Suppression of the coffee-ring effect by shape-dependent capillary interactions. Nature, 2011. 476(7360): p. 308-311.

15. Eral, H.B., D.J.C.M. 't Mannetje, and J.M. Oh, Contact angle hysteresis: a review of fundamentals and applications. Colloid and Polymer Science, 2013. 291(2): p. 247-260.

16. Shimobayashi, S.F., M. Tsudome, and T. Kurimura, Suppression of the coffee-ring effect by sugar-assisted depinning of contact line. Sci Rep, 2018. 8(1): p. 17769.

17. Yang, H., et al., Controlling the Coffee Ring Effect on Graphene and Polymer by Cations. Chinese Physics Letters, 2020. 37(2): p. 028103.

18. Seo, C., et al., Altering the coffee-ring effect by adding a surfactant-like viscous polymer solution. Scientific Reports, 2017. 7(1): p. 500.

19. Talbot, E.L., et al., Control of the Particle Distribution in Inkjet Printing through an Evaporation-Driven Sol-Gel Transition. ACS Applied Materials \& Interfaces, 2014. 6(12): p. 9572-9583.

20. Cui, L., et al., Suppression of the Coffee Ring Effect by Hydrosoluble Polymer Additives. ACS Applied Materials \& Interfaces, 2012. 4(5): p. 2775-2780.

21. Vunain, E., A.K. Mishra, and B.B. Mamba, 1 - Fundamentals of chitosan for biomedical applications, in Chitosan Based Biomaterials Volume 1, J.A. Jennings and J.D. Bumgardner, Editors. 2017, Woodhead Publishing. p. 3-30. 
22. Kim, J.-H., et al., Polymer Transports Inside Evaporating Water Droplets at Various Substrate Temperatures. The Journal of Physical Chemistry C, 2011. 115(31): p. 15375-15383.

23. Erbil, H.Y., G. McHale, and M.I. Newton, Drop evaporation on solid surfaces: Constant contact angle mode. Langmuir, 2002. 18(7): p. 2636-2641.

24. Parsa, M., S. Harmand, and K. Sefiane, Mechanisms of pattern formation from dried sessile drops. Advances in Colloid and Interface Science, 2018. 254: p. 22-47.

25. Larson, R.G., Re-Shaping the Coffee Ring. Angewandte Chemie International Edition, 2012. 51(11): p. 2546-2548.

26. Hu, H. and R.G. Larson, Marangoni Effect Reverses Coffee-Ring Depositions. The Journal of Physical Chemistry B, 2006. 110(14): p. 7090-7094. 